\title{
The evolution of mass profiles of galaxy clusters
}

\author{
A. Biviano* and B. Poggianti ${ }^{\dagger}$ \\ *INAF/Osservatorio Astronomico di Trieste, Italy \\ $\dagger$ INAF/Osservatorio Astronomico di Padova, Italy
}

\begin{abstract}
We determine the average mass profile of galaxy clusters at two different redshifts and compare its evolution with cosmological model predictions. We use two samples of galaxy clusters spanning similar (evolutionary corrected) mass ranges at different redshifts. The sample of low-redshift $(z \simeq 0.0-0.1)$ clusters is extracted from the ESO Nearby Abell Cluster Survey (ENACS) catalog. The sample of high-redshift $(z \simeq 0.4-0.8)$ clusters is mostly made of clusters from the ESO Distant Cluster Survey (EDisCS). We determine the average mass-profiles for these two cluster samples by solving the Jeans equation for hydrostatic equilibrium, using galaxies as tracers. By using two cluster galaxy populations, characterized by the presence and, respectively, absence of emission-lines in their spectra ('ELGs' and 'nELGs' hereafter), we are able to partially break the mass-profile orbital-anisotropy degeneracy.

We find that the mass-profiles of both the nearby and the distant clusters are reasonably well fitted by a Navarro, Frenk $\&$ White (NFW) model. The best-fit values of the NFW concentration parameter are as predicted by cosmological numerical simulations; cluster mass-density profiles become more concentrated with time. The evolution of the number-density profile of nELGs proceeds in the opposite sense, becoming less concentrated with time.

In our analysis we also recover the orbital anisotropy of nELGs and ELGs. We find that in low- $z$ clusters nELGs follow almost isotropic orbits and ELGs have more radially-elongated orbits. In high- $z$ clusters both nELGs and ELGs follow radiallyelongated orbits.

We discuss these results in terms of the predicted secular mass growth of galaxy clusters and the transformation of ELGs into nELGs.
\end{abstract}

Keywords: Galaxies: clusters: general - Galaxies: kinematics and dynamics PACS: $98.65 . \mathrm{Cw}$, 98.62.Ck, 98.62.Dm, 98.80.Es

\section{INTRODUCTION}

The study of the mass distribution within clusters and of its evolution with redshift can provide very useful constraints on the nature of dark matter (DM) [1, 2, 3] and on the formation and evolution of galaxy clusters and their components [4, 5, 6]. CDM cosmological simulations have shown that the mass-density profiles of cosmological halos follow a universal profile [7],

$$
\rho_{N F W} \propto\left(c r / r_{200}\right)^{-1}\left(1+c r / r_{200}\right)^{-2},
$$

parameterized by the concentration parameter $c$ 1 1 . This universal model, named 'NFW' after the initials of its proposers, is characterized by a central density cusp. The NFW model has been shown to fit reasonably well the mass-density profile of nearby clusters [e.g. 8, 9, 10, 11, 12, 13], although the precise form of the profile near the center has been a matter of debate [e.g. 14, 15, 16].

Cosmological simulations predict a mild dependence of $c$ on the halo mass, $M$, and a mild evolution of the $c-M$ relation with redshift, $z$ [e.g. 17, 18]. Observed $c$ values of low- $z$ galaxy systems appear to be somewhat higher than theoretical predictions, but the discrepancy mostly concerns low-mass galaxy systems [18, 19]. Massive galaxy clusters at low- $z$ have the expected concentrations [e.g. 10, 20]. This is true also for clusters at redshift $z \approx 0.3[21]$.

Little is known about the mass-density profiles of galaxy clusters at still higher- $z$. Mass-density profile concentrations have so far been estimated for about a dozen clusters in total at $z \gtrsim 0.5[22,23,24,25,26,27,28,29]$, all via gravitational lensing or deep X-ray observations. Here we report on the determination of the average mass-density profile of 19 clusters at $0.39 \leq z \leq 0.8(\bar{z}=0.56)$, based on the projected phase-space distribution of cluster galaxies, used as tracers of the gravitational potential.

\footnotetext{
${ }^{1}$ The virial radius $r_{200}$ is the radius within which the enclosed average mass density of a cluster is 200 times the critical density. The virial mass $M_{200}$ is the mass enclosed within a sphere of radius $r_{200}$.

The circular velocity is defined from the previous two quantities as $\mathrm{v}_{200}=\left(G M_{200} / r_{200}\right)^{1 / 2}$.
} 
We adopt $H_{0}=70 \mathrm{~km} \mathrm{~s}^{-1} \mathrm{Mpc}^{-1}, \Omega_{m}=0.3, \Omega_{\Lambda}=0.7$ throughout this paper.

\section{THE SAMPLES}

We use two samples of galaxy clusters, one at $\bar{z}=0.56$, the EDisCS sample [30, 31, 32, 33, 34], and another at $\bar{z}=0.07$, the ENACS sample [35, 36], to investigate the evolution of the average cluster mass profile.

We select the 15 EDisCS clusters with velocity dispersion $\sigma_{l o s} \geq 250 \mathrm{~km} \mathrm{~s}^{-1}$, in order to have a more homogeneous data-set in terms of mass. We then add to this sample four clusters from the MORPHS [37, 38], all with masses in the same range covered by the 15 EDisCS clusters. All 19 clusters have sufficiently wide spatial coverage $\left(>0.5 r_{200}\right)$ for the dynamical analysis, as well as homogeneous photometry (which is needed for the determination of the radial incompleteness, see below).

Among the ENACS clusters, we use the 59 ENACS clusters studied in detail by [39, 10, 40].

We identify cluster members by the procedure described in [41], which has been validated on cluster-sized halos extracted from cosmological numerical simulations [41, 42]. We then determine cluster line-of-sight (los) velocity dispersions $\sigma_{l o s}$ by applying the robust biweight estimator to the velocity distributions of selected cluster members [43]. Cluster masses $M_{200}$ are determined from the $\sigma_{l o s}$-estimates using the $\sigma_{l o s}-M_{200}$ relation of [44].

In summary, our data-set consist of 19 distant clusters from $z=0.39$ to $0.80(\bar{z}=0.56)$ with $M_{200}$ masses from 0.7 to $13.6 \times 10^{14} M_{\odot}\left(\right.$ mean: $\left.2.8 \times 10^{14} M_{\odot}\right)$ and 59 nearby clusters from $z=0.03$ to $0.10(\bar{z}=0.07)$ with $M_{200}$ masses from 0.4 to $20.5 \times 10^{14} M_{\odot}$ (mean: $5.9 \times 10^{14} M_{\odot}$ ). When account is taken for the predicted evolution in mass between $z=0.56$ and $z=0.07$ [45, 46] the two samples are found to contain halos of similar (evolutionary-corrected) masses at two different cosmic epochs.

Determination of the average cluster mass profile requires stacking together all the clusters in each of the two samples. Stacking is done by scaling the projected clustercentric galaxy distances, $R$, by cluster virial radii, $r_{200}$, and the los galaxy velocities (in the cluster rest frame), $\mathrm{v}_{r f} \equiv(\mathrm{v}-\overline{\mathrm{v}}) /(1+\overline{\mathrm{v}} / c)$, by cluster circular velocities, $\mathrm{v}_{200}$. In such a way we avoid mixing up the virialized regions of the more massive clusters with the unvirialized, external regions of the less massive ones. Since cluster mass profiles are expected to depend very mildly on cluster mass [e.g. 7, 47], the shape of the stacked cluster mass-profile is expected to be similar to the average shape of the individual cluster mass profiles.

The center of each cluster is defined to be the position of its X-ray surface-brightness peak, when available, or the position of its brightest cluster galaxy otherwise. We only use galaxies in the radial range $0.05 \leq R / r_{200} \leq 1$ in the dynamical analysis. At $R / r_{200}<0.05$ the analysis is unreliable because of the uncertainty in the position of the cluster center, and at $R>r_{200}$ the analysis is unreliable because dynamical relaxation is not guaranteed outside the virial region. In this radial range the high- $z$ (low- $z$ ) stacked cluster contains 556 (respectively 2566) galaxies.

We identify two populations of tracers: nELGs, the galaxies without emission lines in their spectra, and ELGs, galaxies with emission lines. More specifically, we classify ELGs the EDisCS galaxies with an [OII] equivalent width $\geq 3 \AA$ or with any other line in emission [32] and the MORPHS galaxies with a spectral type different from ' $k$ ', ' $k+a$ ', and ' $a+k$ ' [38]. We refer to [35] for the ELG classification of ENACS galaxies. The fraction of ELGs among the galaxies selected for the dynamical analysis is $47 \%$ in the high- $z$ sample, and only $13 \%$ in the low- $z$ sample, a difference that reflects the evolution of the properties of cluster galaxies [37, 38, 32].

The samples are not spectroscopically complete to a given magnitude. This is not a problem for the dynamical analysis as far as the incompleteness does not depend on radius. The spectroscopic incompleteness is indeed independent on radius for the ENACS [36]. On the other hand, for the high- $z$ cluster sample we need to weigh galaxies according to their radial positions when determining the galaxy number density profiles. The weighting method is described in [32].

In constructing the galaxy number density profiles, we also need to assign different weights to the contributions of different clusters to the number counts in a given radial bin. This is because each cluster contributes galaxies to the stacked sample only out to a limiting radius, which is defined by the observational set-up. Hence, while at small radii all clusters contribute, at large radii we need to correct for those clusters that have not been sampled. The correction method is similar to the one described in [48] - see [39] and [10] for applications to the ENACS. 

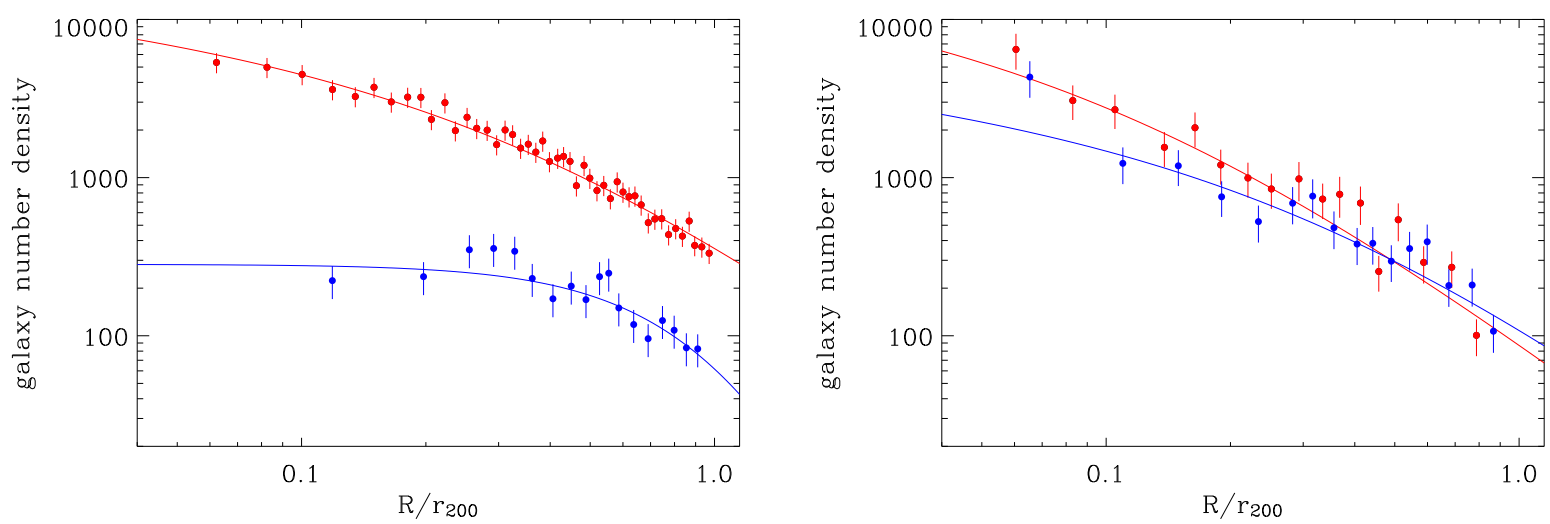

FIGURE 1. Left panel: The projected number density profiles, $N(R)$, of the nELGs (red) and ELGs (blue) in the low- $z$ stacked cluster. Right panel: same as left panel, but for the high- $z$ stacked cluster. Solid lines represent best-fit models to the data, i.e. projected NFW models for nELGs and high- $z$ ELGs, and the core model for low- $z$ ELGs.
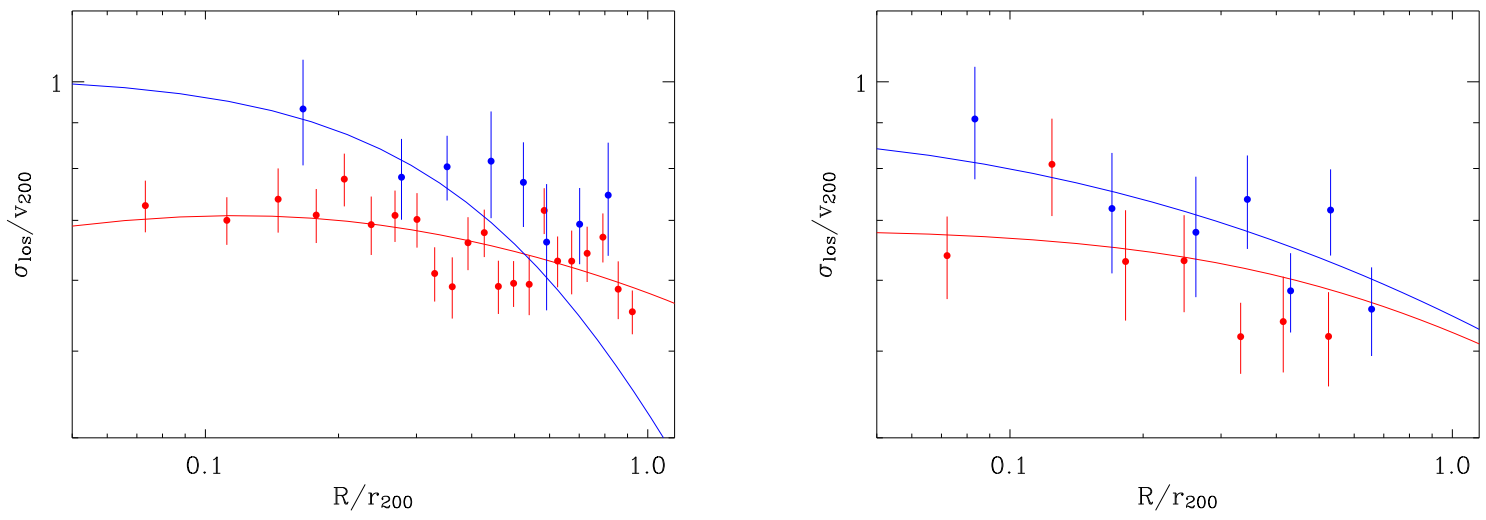

FIGURE 2. Left panel: The los velocity dispersion profiles $\sigma_{l o s}(R)$, of the nELGs (red) and ELGs (blue) in the low- $z$ stacked cluster. Right panel: same as left panel, but for the high- $z$ stacked cluster. Solid lines represent best-fit models to the data, i.e. a $c=4.0 \mathrm{NFW}$ mass model, $a / r_{200}=3.6$ (respectively $a / r_{200}=1.2$ ) OM velocity-anisotropy model for low- $z$ nELGs (respectively, ELGs), and a $c=3.2 \mathrm{NFW}$ mass model, $a / r_{200}=0.01$ MŁ velocity-anisotropy model for high- $z$ nELGs and ELGs.

\section{THE METHOD}

The method we adopt for the dynamical analysis of our two stacked clusters is based on the standard sphericallysymmetric Jeans analysis 4992 . In this analysis, the observables are the galaxy number density profile $N(R)$ and the los velocity dispersion profile $\sigma_{l o s}(R)$. These are displayed in Figures 1 and 2 , respectively, separately for the two cluster samples and for the two cluster galaxy populations.

$N(R)$ is uniquely related to the 3-D galaxy number density profile $v(r)$ via the Abel inversion equation. The other observable, $\sigma_{l o s}(R)$, is then uniquely determined if both the cluster mass profile, $M(r)$, and the cluster velocity anisotropy profile, $\beta(r)$, are known [50, 51]. The velocity anisotropy profile $\beta(r)$ is

$$
\beta(r) \equiv 1-\frac{<\mathrm{v}_{\mathrm{t}}^{2}>}{<\mathrm{v}_{\mathrm{r}}^{2}}
$$

\footnotetext{
${ }^{2}$ Spherical symmetry is imposed by the stacking procedure, which is irrespective of the individual cluster position angles
} 
where $\left\langle\mathrm{v}_{\mathrm{t}}^{2}\right\rangle,\left\langle\mathrm{v}_{\mathrm{r}}^{2}\right\rangle$ are the mean squared tangential and radial velocity components, which reduce to $\sigma_{t}^{2}$ and $\sigma_{r}^{2}$, respectively, in the absence of bulk motions and net rotation (as we assume in the present analysis).

We adopt parameterized model representations of $M(r)$ and $\beta(r)$ and determine the best-fit parameters of these models by comparing the observed $\sigma_{l o s}(R)$ profile with the predicted one, using the $\chi^{2}$ statistics and the uncertainties on the observed profile. In order to reduce the so-called "mass-anisotropy" degeneracy which plagues these kinds of analyses [see, e.g., 52, 21, 53] we adopt the method recently suggested by [54]. Namely, we consider two independent tracers of the same gravitational potential, nELGs and ELGs, and determine the best-fit parameters of $M(r)$ and $\beta(r)$ by a joint $\chi^{2}$-analysis of the best-fits to the $\sigma_{l o s}(R)$ profiles of the two populations. Clearly $M(r)$ must be the same for both tracers, but $\beta(r)$ can in principle be different, so the degeneracy is only partially broken; however the constraints on the dynamics of the system are significantly stronger than when using a single tracer.

Our choice of the $M(r)$ and $\beta(r)$ models is driven by the results of the analysis of cluster-sized halos extracted from cosmological numerical simulations. We adopt the NFW model, eq. (1), parameterized by the concentration $c$, for the mass-density profile and also for the galaxy number-density profile 3 , but we allow different concentrations for the galaxy and the mass distributions. Only when the projected-NFW model does not provide an acceptable fit to $N(R)$, we consider an alternative model,

$$
N \propto\left[1+\left(R / R_{c}\right)^{2}\right]^{-\alpha} .
$$

We refer to this model[56] as the 'core' model, since it is characterized by a central constant density. [57]

We consider two models for the velocity-anisotropy profile $\beta(r)$. One is the Mamon-Łokas ('MŁ' hereafter) model

$$
\beta=0.5 r /(r+a),
$$

and the other is the Osipkov-Merritt ('OM' hereafter) model [58, 59]

$$
\beta=r^{2} /\left(r^{2}+a^{2}\right)
$$

Both the MŁ and the OM models depend on just one free parameter, the anisotropy radius, $a$, which marks the transition from the central region, where $\beta \approx 0$ and the galaxy orbits are isotropic, to the external region, where $\beta>0$ and the galaxy orbits become increasingly radial.

\section{RESULTS}

\section{The nearby cluster sample}

The nELG $N(R)$ is best-fitted by a (projected) NFW profile with $c=2.4$. A core model is required to fit the ELG $N(R)$ which avoid the central cluster region, with best-fit parameter values $R_{c} / r_{200}=1.28$ and $\alpha=3.2$. The best-fit models are displayed in Figure 1 (left panel). Abel-inversion of the $N(R)$ best-fitting models provides the 3-D number density profiles $v(r)$.

The best-fit $c$ parameter of the stacked cluster NFW mass-density profile is determined by a joint $\chi^{2}$ fit to the observed $\sigma_{l o s}$-profiles of the nELGs and ELGs. The best-fit solution is obtained when the OM model is adopted for the velocity anisotropy profiles of the two galaxy populations. The best-fit value of the NFW concentration parameter is $c=4.0_{-1.3}^{+2.3}$ (90\% confidence levels, c.l. in the following), in agreement with [10]. The $\chi^{2}$ vs. $c$ solution is displayed in Figure 3 (green curve).

Using this solution for the cluster mass profile we obtain the best-fit $\beta(r)$ OM-model parameters $a / r_{200}=3.6_{-1.6}^{+13.4}$ and $a / r_{200}=1.2_{-0.4}^{+1.2}$ for the nELG and ELG populations, respectively $(90 \%$ c.l.). These $\beta(r)$ profiles are shown in the left panel of Figure 4 [where we actually display $\sigma_{r} / \sigma_{t} \equiv(1-\beta)^{-1 / 2}$ ]. The best-fit $\beta(r)$ solutions indicate that nELGs follow isotropic orbits within the cluster virial region, and that ELG orbits are isotropic near the center but become increasingly radial in the outer cluster regions. These results are in agreement with [40].

In Figure 2 (left panel) we display the observed los velocity dispersion profiles, and the best-fit obtained via the Jeans analysis.

\footnotetext{
${ }^{3}$ More precisely, we fit the projected NFW profile [55] to $N(R)$.
} 


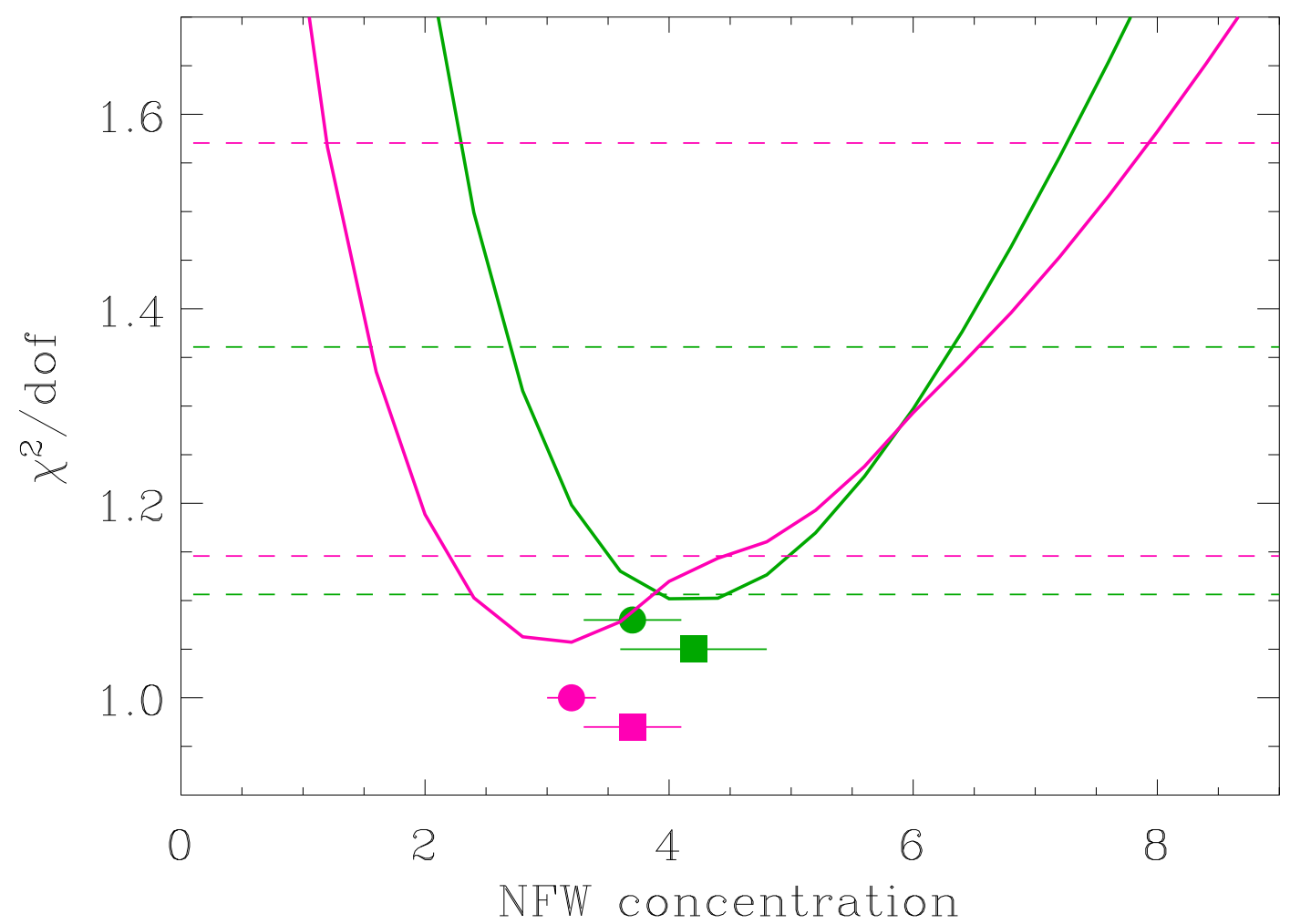

FIGURE 3. The values of $\chi^{2}$ per degree of freedom obtained from the joint fit to the $\sigma_{l o s}(R)$ profiles of nELGs and ELGs for different values of the $c$ parameter of the NFW mass-profile model. The green and pink curves are for the low- $z$, and, respectively, the high- $z$ stacked cluster. The two dashed lines indicate the $68 \%$ and $90 \%$ c.l. Symbols are theoretical predictions for the concentrations of the mass-density profiles of CDM halos with the same masses and redshifts as the clusters of our samples (squares: [17], dots: [18]; green: low- $z$, pink: high-z). The symbol error-bars are obtained by considering the 1- $\sigma$ range in the distributions of cluster masses and average redshifts.
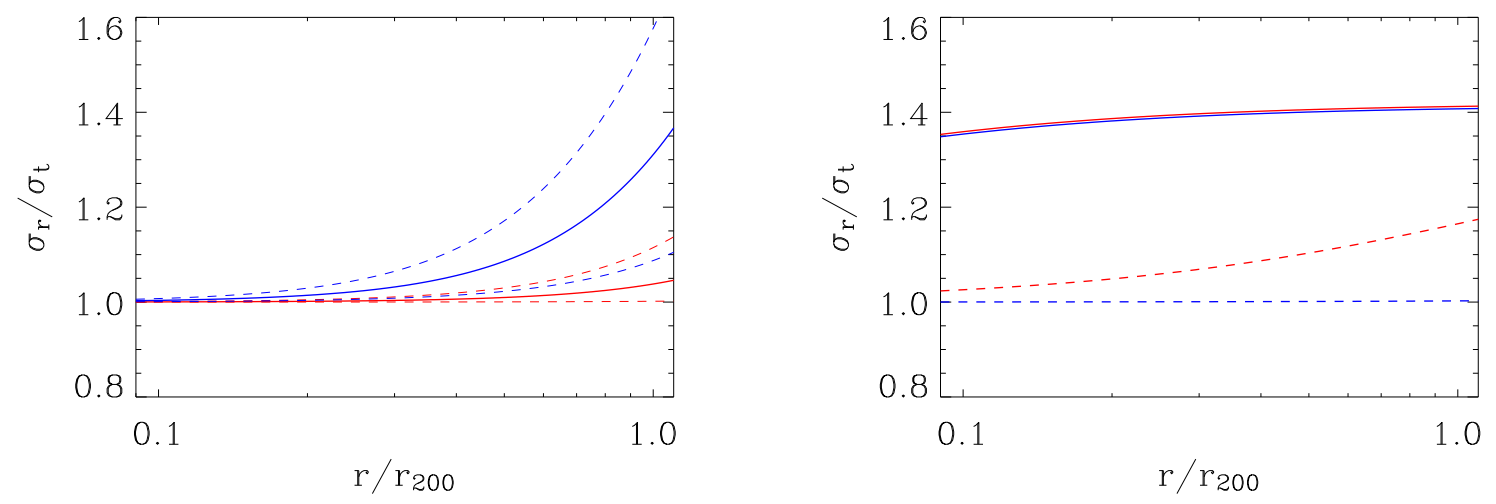

FIGURE 4. Left panel: Best-fit velocity-anisotropy profile $\sigma_{r} / \sigma_{t} \equiv(1-\beta)^{-1 / 2}$ for nELGs (red curve) and ELGs (blue curve) in the low- $z$ stacked cluster. Dashed curves indicate $90 \%$ c.l. Right panel: same as left panel, but for the high- $z$ stacked cluster. In this case the best-fit solutions are at the lower-limit of the interval considered in the $\chi^{2}$ minimization analysis, so only the lower c.l. to $\beta(r)$ are shown. 


\section{The distant cluster sample}

Projected NFW models provide a good fit to the $N(R)$ of nELGs and ELGs with best-fit parameters $c=7.5$ and $c=2.7$, respectively (see the right panel of Figure1). Abel-inversion of these best-fitting models then provide the 3-D number density profiles $v(r)$ that we use in the dynamical analysis.

The joint best-fit to the los $\sigma_{l o s}$-profiles of nELGs and ELGs is obtained when the MŁ $\beta(r)$ model is adopted. The mass profile best-fit concentration value is $c=3.2_{-2.0}^{+4.6}$ (90\% c.l.). The $\chi^{2}$ vs. $c$ solution is displayed in Figure 3 (pink curve). Using this solution for the stacked cluster mass profile, we find that the best-fit value of the MŁ $\beta(r)$ model parameter $a$, is the same for the nELGs and for the ELGs, $a / r_{200}=0.01$, at the lower limit of the $a$-range considered in the $\chi^{2}$ minimization analysis. The solution is only poorly constrained, $a<0.9 r_{200}$ and $a \leq 10.0 r_{200}$, for the nELGs and the ELGs, respectively. The best-fit velocity-anisotropy profiles are shown in Figure 4(right panel). Both cluster galaxy populations are characterized by radially anisotropic orbits, although isotropy cannot be formally excluded for the ELGs, given the uncertainties.

The observed $\sigma_{l o s}$-profiles are shown in the right panel of Figure 2, together with the best-fit solutions from the Jeans analysis.

\section{DISCUSSION AND PERSPECTIVES}

Using cluster galaxies as tracers of the gravitational potential, we have solved the Jeans analysis for the dynamical equilibrium of a spherical system, and determined the average mass-density profiles of galaxy clusters at $z \simeq 0.1$ and at $z \simeq 0.6$. These mass-density profiles are well described by NFW models with $c$ values which decrease with increasing $z$. The best-fit $c$ values are in agreement with the theoretical predictions of $\Lambda$ CDM models. This can be seen in Figure 3 where we display our best-fit results together with the theoretically predicted mean $c$ values. These are determined by using the masses and redshifts of the clusters in our two samples and applying the theoretical $c=c(M, z)$ relations of [17] and [18].

Our result appears to disagree with the conclusions of [18] who claim that the observed $c$ values of clusters and groups are significantly above the theoretically expected ones for group- and cluster-sized cosmological halos. However most of the claimed discrepancy is for low-mass galaxy systems, which are not covered by the present analysis. Preliminary dynamical analyses of the mass-density profiles of low- $z$ groups [19], done with similar techniques as the one employed here, do indicate a discrepancy in the direction reported by [18].

An additional result of our dynamical analysis is the determination of galaxy orbits in clusters at low- $z$ and high- $z$. We find that these orbits become more isotropic with time. While low- $z$ cluster nELGs have nearly isotropic orbits, high $-z$ cluster $n E L G s$ move on radially elongated orbits, and so do both low $-z$ and high $-z$ ELGs (compare the left-hand and right-hand panels of Figure 4).

Orbital isotropization might result from the hierarchical accretion process of clusters [60] which undergo an initial, fast accretion phase, followed by a slower, smoother accretion phase [46]. During the fast accretion phase clusters are subject to rapid variations of their gravitational potential [61, 62, 63], and these are capable of isotropizing galaxy orbits [64, 65, 66, 67, 46]. The end of the fast accretion phase for cluster-sized halos occurs at $z \approx 0.4$ [46], hence it is not over yet for most of the clusters of our high- $z$ sample. This can explain why the orbits of high- $z$ cluster galaxies have not become isotropic yet. At lower- $z$, galaxies that have entered the cluster environment lately may experience only a slower orbital isotropization process, probably caused by ram-pressure [68]. Cluster ELGs are in this situation, while low- $z$ cluster $n E L G s$ have developed isotropic orbits already because they were accreted much earlier than ELGs.

The different relative fraction of nELGs and ELGs in low- and high- $z$ clusters suggest that ELGs gradually transform into nELGs [32]. Since ELGs have a wider spatial distribution in clusters than nELGs, as ELGs join the nELG population, the global nELG spatial distribution become less concentrated. This is indeed observed in our data-sets (compare the red curves in the left and right panels of Figure 1).

The results presented here are still preliminary, as their statistical significance is not very strong given the rather limited size of the data-set for high- $z$ cluster galaxies. Substantial improvement in the understanding of the accretion and internal dynamics history of galaxy clusters requires much better statistics. In this sense, the proposed ESA mission "EUCLID' 4 for the measurement of Dark Energy is extremely promising. As of this writing, the EUCLID mission

4 http://sci.esa.int/science-e/www/area/index.cfm?fareaid=102 
proposal considers a $20,000 \mathrm{deg}^{2}$ spectroscopic survey with a near-IR slitless spectrometer down to a sensitivity level of $4 \times 10^{-16} \mathrm{erg} \mathrm{cm}^{-2} \mathrm{~s}^{-1}$ for emission-lines, with a $1 / 3$ sampling rate. Such a survey should return $\simeq 10,000$ clusters, each with $>20$ cluster members with measured redshifts, at $0.5 \leq z \leq 0.8$. By stacking together clusters of similar mass it should be possible to constrain not only the evolution of the average cluster mass-density profile to an accuracy of $\sim 3 \%$ in $c$, but also to measure the redshift evolution of the $c=c(M)$ relation. With the same sample it will also be possible to determine the evolution of the ELG orbits in clusters, and to infer the history of cluster mass accretion.

\section{ACKNOWLEDGMENTS}

We acknowledge useful discussions with Giuseppina Battaglia, Alfonso Cavaliere, Florence Durret, Marisa Girardi, and Gary Mamon. This research has been financially supported from the National Institute for Astrophysics through the PRIN-INAF scheme. This research has made use of NASA's Astrophysics Data System.

\section{REFERENCES}

1. D. N. Spergel, and P. J. Steinhardt, Physical Review Letters 84, 3760-3763 (2000).

2. M. Meneghetti, N. Yoshida, M. Bartelmann, L. Moscardini, V. Springel, G. Tormen, and S. D. M. White, MNRAS 325, 435-442 (2001).

3. D. Reed, F. Governato, L. Verde, J. Gardner, T. Quinn, J. Stadel, D. Merritt, and G. Lake, MNRAS 357, 82-96 (2005).

4. V. Springel, S. D. M. White, G. Tormen, and G. Kauffmann, MNRAS 328, 726-750 (2001).

5. A. A. El-Zant, Y. Hoffman, J. Primack, F. Combes, and I. Shlosman, ApJ 607, L75-L78 (2004).

6. L. Gao, G. De Lucia, S. D. M. White, and A. Jenkins, MNRAS 352, L1-L5 (2004).

7. J. F. Navarro, C. S. Frenk, and S. D. M. White, ApJ 490, 493-508 (1997).

8. A. Biviano, and M. Girardi, ApJ 585, 205-214 (2003).

9. K. Rines, M. J. Geller, M. J. Kurtz, and A. Diaferio, AJ 126, 2152-2170 (2003).

10. P. Katgert, A. Biviano, and A. Mazure, ApJ 600, 657-669 (2004).

11. E. Pointecouteau, M. Arnaud, and G. W. Pratt, $A \& A$ 435, 1-7 (2005).

12. A. Biviano, and P. Salucci, $A \& A$ 452, 75-81 (2006).

13. E. L. Łokas, R. Wojtak, S. Gottlöber, G. A. Mamon, and F. Prada, MNRAS 367, 1463-1472 (2006).

14. B. Moore, T. Quinn, F. Governato, J. Stadel, and G. Lake, MNRAS 310, 1147-1152 (1999).

15. E. Hayashi, J. F. Navarro, C. Power, A. Jenkins, C. S. Frenk, S. D. M. White, V. Springel, J. Stadel, and T. R. Quinn, MNRAS 355, 794-812 (2004).

16. J. Diemand, M. Zemp, B. Moore, J. Stadel, and C. M. Carollo, MNRAS 364, 665-673 (2005).

17. L. Gao, J. F. Navarro, S. Cole, C. S. Frenk, S. D. M. White, V. Springel, A. Jenkins, and A. F. Neto, MNRAS 387, 536-544 (2008).

18. A. R. Duffy, J. Schaye, S. T. Kay, and C. Dalla Vecchia, MNRAS 390, L64-L68 (2008).

19. A. Biviano, arXiv:0811.3535, 2008.

20. K. Rines, and A. Diaferio, AJ 132, 1275-1297 (2006).

21. R. P. van der Marel, J. Magorrian, R. G. Carlberg, H. K. C. Yee, and E. Ellingson, AJ 119, 2038-2052 (2000).

22. S. W. Allen, R. W. Schmidt, and A. C. Fabian, MNRAS 335, 256-266 (2002).

23. H. Hoekstra, M. Franx, K. Kuijken, and P. G. van Dokkum, MNRAS 333, 911-922 (2002).

24. M. J. Jee, R. L. White, N. Benítez, H. C. Ford, J. P. Blakeslee, P. Rosati, R. Demarco, and G. D. Illingworth, ApJ 618, 46-67 (2005).

25. L. M. Voigt, and A. C. Fabian, MNRAS 368, 518-533 (2006).

26. B. J. Maughan, C. Jones, L. R. Jones, and L. Van Speybroeck, ApJ 659, 1125-1137 (2007).

27. J. Rzepecki, M. Lombardi, P. Rosati, A. Bignamini, and P. Tozzi, A\&A 471, 743-752 (2007).

28. R. W. Schmidt, and S. W. Allen, MNRAS 379, 209-221 (2007).

29. A. Halkola, H. Hildebrandt, T. Schrabback, M. Lombardi, M. Bradač, T. Erben, P. Schneider, and D. Wuttke, A\&A 481, 65-77 (2008).

30. C. Halliday, B. Milvang-Jensen, S. Poirier, B. M. Poggianti, P. Jablonka, A. Aragón-Salamanca, R. P. Saglia, G. De Lucia, R. Pelló, L. Simard, D. I. Clowe, G. Rudnick, J. J. Dalcanton, S. D. M. White, and D. Zaritsky, A\&A 427, 397-413 (2004).

31. S. D. M. White, D. I. Clowe, L. Simard, G. Rudnick, G. De Lucia, A. Aragón-Salamanca, R. Bender, P. Best, M. Bremer, S. Charlot, J. Dalcanton, M. Dantel, V. Desai, B. Fort, C. Halliday, P. Jablonka, G. Kauffmann, Y. Mellier, B. Milvang-Jensen, R. Pelló, B. Poggianti, S. Poirier, H. Rottgering, R. Saglia, P. Schneider, and D. Zaritsky, A\&A 444, 365-379 (2005).

32. B. M. Poggianti, A. von der Linden, G. De Lucia, V. Desai, L. Simard, C. Halliday, A. Aragón-Salamanca, R. Bower, J. Varela, P. Best, D. I. Clowe, J. Dalcanton, P. Jablonka, B. Milvang-Jensen, R. Pello, G. Rudnick, R. Saglia, S. D. M. White, and D. Zaritsky, ApJ 642, 188-215 (2006).

33. V. Desai, J. J. Dalcanton, A. Aragón-Salamanca, P. Jablonka, B. Poggianti, S. M. Gogarten, L. Simard, B. Milvang-Jensen, G. Rudnick, D. Zaritsky, D. Clowe, C. Halliday, R. Pelló, R. Saglia, and S. White, ApJ 660, 1151-1164 (2007). 
34. B. Milvang-Jensen, S. Noll, C. Halliday, B. M. Poggianti, P. Jablonka, A. Aragón-Salamanca, R. P. Saglia, N. Nowak, A. von der Linden, G. De Lucia, R. Pelló, J. Moustakas, S. Poirier, S. P. Bamford, D. I. Clowe, J. J. Dalcanton, G. H. Rudnick, L. Simard, S. D. M. White, and D. Zaritsky, A\&A 482, 419-449 (2008).

35. P. Katgert, A. Mazure, J. Perea, R. den Hartog, M. Moles, O. Le Fevre, P. Dubath, P. Focardi, G. Rhee, B. Jones, E. Escalera, A. Biviano, D. Gerbal, and G. Giuricin, $A \& A$ 310, 8-30 (1996).

36. P. Katgert, A. Mazure, R. den Hartog, C. Adami, A. Biviano, and J. Perea, A\&As 129, 399-412 (1998).

37. A. Dressler, I. Smail, B. M. Poggianti, H. Butcher, W. J. Couch, R. S. Ellis, and A. J. Oemler, ApJS 122, 51-80 (1999).

38. B. M. Poggianti, I. Smail, A. Dressler, W. J. Couch, A. J. Barger, H. Butcher, R. S. Ellis, and A. J. Oemler, ApJ 518, 576-593 (1999).

39. A. Biviano, P. Katgert, T. Thomas, and C. Adami, $A \& A$ 387, 8-25 (2002).

40. A. Biviano, and P. Katgert, $A \& A$ 424, 779-791 (2004).

41. A. Biviano, G. Murante, S. Borgani, A. Diaferio, K. Dolag, and M. Girardi, A\&A 456, 23-36 (2006).

42. R. Wojtak, E. L. Łokas, G. A. Mamon, S. Gottlöber, F. Prada, and M. Moles, A\&A 466, 437-449 (2007).

43. T. C. Beers, K. Flynn, and K. Gebhardt, AJ 100, 32-46 (1990).

44. J.-C. Mauduit, and G. A. Mamon, $A \& A$ 475, 169-185 (2007).

45. C. Adami, A. Biviano, F. Durret, and A. Mazure, $A \& A$ 443, 17-27 (2005).

46. A. Lapi, and A. Cavaliere, ApJ 692, 174-186 (2009).

47. K. Dolag, M. Bartelmann, F. Perrotta, C. Baccigalupi, L. Moscardini, M. Meneghetti, and G. Tormen, A\&A 416, 853-864 (2004).

48. M. R. Merrifield, and S. M. Kent, $A J$ 98, 351-366 (1989).

49. J. Binney, and S. Tremaine, Galactic dynamics, Princeton, NJ, Princeton University Press, 1987, 747 p., 1987.

50. R. P. van der Marel, MNRAS 270, 271-297 (1994).

51. J. Binney, and G. A. Mamon, MNRAS 200, 361-375 (1982).

52. D. Merritt, ApJ 313, 121-135 (1987).

53. E. L. Łokas, and G. A. Mamon, MNRAS 343, 401-412 (2003).

54. G. Battaglia, A. Helmi, E. Tolstoy, M. Irwin, V. Hill, and P. Jablonka, ApJl 681, L13-L16 (2008).

55. M. Bartelmann, $A \& A$ 313, 697-702 (1996).

56. A. Cavaliere, and R. Fusco-Femiano, $A \& A$ 70, 677-+ (1978).

57. G. A. Mamon, and E. L. Łokas, MNRAS 363, 705-722 (2005).

58. L. P. Osipkov, Soviet Astronomy Letters 5, 42-44 (1979).

59. D. Merritt, MNRAS 214, 25P-28P (1985).

60. S. P. D. Gill, A. Knebe, B. K. Gibson, and M. A. Dopita, MNRAS 351, 410-422 (2004).

61. A. Manrique, A. Raig, E. Salvador-Solé, T. Sanchis, and J. M. Solanes, ApJ 593, 26-37 (2003).

62. S. Peirani, F. Durier, and J. A. de Freitas Pacheco, MNRAS 367, 1011-1016 (2006).

63. M. Valluri, I. M. Vass, S. Kazantzidis, A. V. Kravtsov, and C. L. Bohn, ApJ 658, 731-747 (2007).

64. M. Hénon, Annales d'Astrophysique 27, 83-+ (1964).

65. D. Lynden-Bell, MNRAS 136, 101-+ (1967).

66. H. E. Kandrup, and C. Siopis, MNRAS 345, 727-742 (2003).

67. D. Merritt, New York Academy Sciences Annals 1045, 3 (2005).

68. K. Dolag, S. Borgani, G. Murante, and V. Springel, MNRAS 399, 497-514 (2009). 